\title{
Deployment of a wearable biosensor system in the emergency department: a technical feasibility study
}

\author{
Kristen Miller \\ Brigham and Women's Hospital \\ Kmiller40@une.edu \\ Christopher W Baugh \\ Brigham and Women's Hospital \\ cbaugh@bwh.harvard.edu \\ Peter R Chai \\ Brigham and Women's Hospital \\ pchai@,bwh.harvard.edu
}

\author{
Mohammad Adrian Hasdianda \\ Brigham and Women's Hospital \\ mhasdianda@bwh.harvard.edu
}

\author{
Shreya Divatia \\ Philips Connected Sensing \\ Shreya.divatia@philips.com
}

\author{
Guruprasad D Jambaulikar \\ Brigham and Women's Hospital \\ gjambaulikar@bwh.harvard.edu \\ Edward W Boyer \\ Brigham and Women's Hospital \\ eboyer@bwh.harvard.edu
}

\begin{abstract}
Wearable devices to detect changes in health status are increasingly adopted by consumers, yet hospitals remain slow to assimilate these devices into clinical practice. Despite the clear benefits of capturing clinical information in acutely ill patients, such technology remains difficult to implement in emergency medicine. To improve adoption, barriers must first be removed. In our technical feasibility and acceptability trial, we studied the deployment of a wearable wireless biosensor that collects physiological data. We enrolled 44 adult patients receiving care in an emergency department observation unit. After we consented patients for participation, we applied biosensors to their chest and collected basic demographic and clinical information. We then collected biosensor data on an isolated system and measured patient experience via an exit survey. Throughout this process we documented and studied technical challenges. Overall, the technology was feasible to deploy in the emergency department observation unit and was acceptable to participants. Such technologies have tremendous future operational and clinical implications in settings ranging from emergency to home-care.
\end{abstract}

\section{Introduction}

Increased use of heart rate monitors, smartphone health apps and cutaneous wearable monitors provide unobtrusive, important insights into health conditions. ${ }^{1}$ Paired devices and companion smartphone apps can detect rhythm changes in individuals with atrial fibrillation, measure adherence to oral medications and track changes in spirometry in individuals with lung disease. ${ }^{2-5}$ Despite successful demonstration projects that indicate the feasibility of using wearable devices to measure vital sign parameters, there has been minimal adoption of wearable devices for clinical use in the emergency department. $^{6}$

Emergency departments face rising volumes and increasingly ill patients. In acute settings, understanding the context prior to the presentation of a critically ill patient can help make a diagnosis while providing important information that may guide medical management. ${ }^{6,7}$ Unobtrusive wearable devices have the potential to change not only the management of patients in the emergency department, but also practices in patient throughput and decisions to admit or discharge patients through more frequent monitoring and under ambulatory conditions. ${ }^{8}$

While some investigations suggest that capturing these real-time biophysiologic changes may alter certain health decisions (e.g., detection of arrhythmia may alter the decision to initiate anticoagulation pharmacotherapy), few hospitals have consistently integrated wearable devices into their inpatient practice. ${ }^{9}$ The delay in introduction of wearable devices in the hospital setting is likely due to significant information security and wireless data transmission capability challenges that limit integration with hospital data solution systems.

Wireless biosensors that capture important vital sign information may improve detection of adverse events in the outpatient setting and may serve as a vehicle to conduct traditional inpatient care at home in the setting of a "home hospital" system. ${ }^{10}$ There is 
additional benefit of translating the experience of wearable biosensors from the outpatient setting into the hospital, especially the emergency department. Wearable biosensors can improve a hospital's capacity to conduct telemetry in non-traditional settings, provide safer quality care, and improve the patient hospital experience. Despite the benefits of developing and using wearable biosensors in the hospital, there are significant barriers that must be overcome prior to successful deployment of these devices. In this manuscript, we discuss our experience with information technology challenges, biosensor design considerations and human factors issues that affect the successful deployment of a wearable biosensor system.

\section{Materials and Methods}

We conducted a technical feasibility trial that deployed a prototype wireless wearable biosensor in the emergency department observation unit (EDOU) of an urban, academic, quaternary care center. Our emergency department has an annual volume of 63,000 patients per year, $5-10 \%$ of which receive care in an integrated EDOU annually. The EDOU is designed to function like a short-stay hospital unit and manages patients who have projected hospital stays of up to 48 hours. The intention of the unit is to provide additional time beyond a typical emergency department visit for additional diagnostic and therapeutic interventions for patients who may otherwise not require an inpatient admission in our hospital. Our investigation was approved by the Partners Healthcare Institutional Review Board. We enrolled adult emergency department patients who were assigned by the primary clinical team to the EDOU.

\subsection{Wearable Biosensor and Connectivity}

We deployed a single-use, cutaneous, chest-worn investigational biosensor that collects physiological data and contextual parameters consisting of heart rate, respiratory rate, activity level, activity type, and posture (Philips Connecting Sensing, Cambridge, MA) (Figure 1). ${ }^{8}$ The biosensor is made of disposable foam with a hydrocolloid adhesive that houses a lithium coin battery, Bluetooth Low Energy (BLE) radio, accelerometer, and two hydrogel-based electrocardiogram (ECG) electrodes. An advanced hydrocolloid adhesive allows the biosensor to adhere to human skin and is designed to minimize irritation while maximizing adhesion. A previous version of this biosensor had been demonstrated to record respiratory rate in emergency department patients. ${ }^{8}$ Iterative refinements of the original version of the wearable biosensor had been integrated into the novel version used in this investigation.

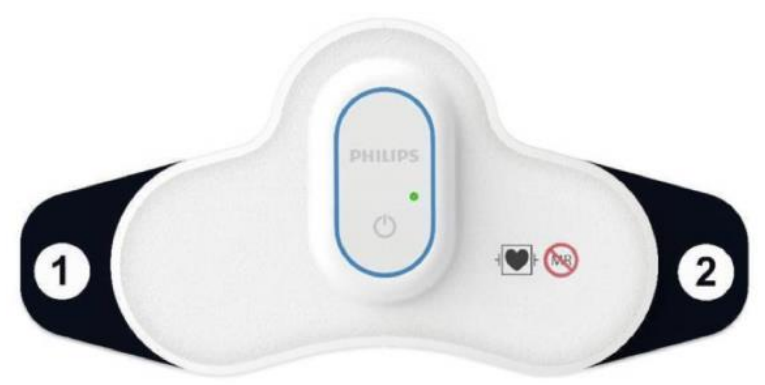

Figure 1: A cutaneous, wearable biosensor, the Philips BX100.

The wireless wearable biosensors are connected to a Wi-Fi network in the EDOU via two BLE routers and a wireless access point installed in the ceiling of the EDOU. We elected to utilize a separate BLE network with wireless access point to prevent the wearable sensors from communicating with our hospital network. This step ensured that critical clinical patient data could not be interdicted by malicious software using the biosensor as a bridge. Proprietary software (access controller software and business logic software) installed on the physical servers at the EDOU nurses' station provides management of connected biosensors and facilitates communication with the clinical research database. A clinical research mobile application installed on a tablet computer provides a clinician interface for patient assignment and review of biosensor data.

\subsection{Eligibility and Consent}

English speaking patients, ages 18 and older, receiving care in the EDOU were eligible for this study. We enrolled patients between April and June of 2019. We identified potential participants through passive screening of the electronic medical record (EMR). The research team approached potentially eligible participants and confirmed eligibility criteria. We obtained written informed consent from interested participants.

\subsection{Study Procedures}

Consented patients underwent a skin assessment and skin preparation procedure (similar to routine ECG lead preparation) prior to biosensor placement. We collected a brief medical history and demographic 
information prior to biosensor placement and patients received a wristband to indicate study participation. Enrollment began at the time of biosensor placement. We placed biosensors on the upper left chest and patients wore the device for the full duration of their EDOU stay. If a patient's stay in the EDOU exceeded 24 hours, study staff performed daily device adhesion and skin assessments. During study participation, patients were free to move in and out of the EDOU without limitation. Before patient discharge from the EDOU, study staff removed the biosensor and performed a post-removal skin assessment. Participants were asked to complete the Participant Experience Questionnaire, a seven-question survey aimed to rate the overall experience and level of comfort of wearing the biosensor. Participants who successfully completed all protocol-required activities were compensated with a \$50 gift card.

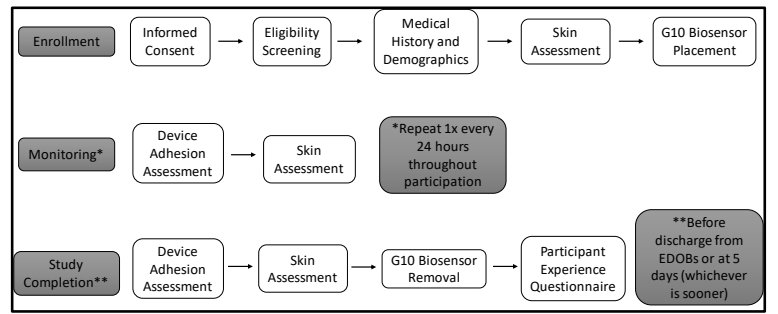

Figure 2: Study schema

\subsection{Data Collection and Analysis}

The research team collected and recorded clinical data team on paper case report forms (CRFs) and then transcribed into the Datatrak Electronic Data Capture (EDC) system. ${ }^{11}$ Datatrak is a secure, web-based EDC system that allows for capture and delivery of data with advanced reporting and optimized workflow.

We collected basic demographic information on all study participants and calculated basic descriptive statistics.

We collected biosensor data in a separate research database to enable analysis related to the technology deployment, system, and biosensor performance.

\section{Results}

During the study period, 290 patients were screened, and 52 individuals met eligibility criteria and were approached by a member of the research team regarding participation. Of those, $86.5 \%(n=45)$ consented to participate in the study. Of the 45 patients consented to the study, 44 patients were enrolled and 40 patients completed all protocol-required study activities. Four participants were unable to complete the study due to incomplete data (e.g., biosensor applied for less than 12 hours, participant requested withdrawal from the study, and enrolled participant but no biosensor applied given an alternative triage decision outside of EDOU.

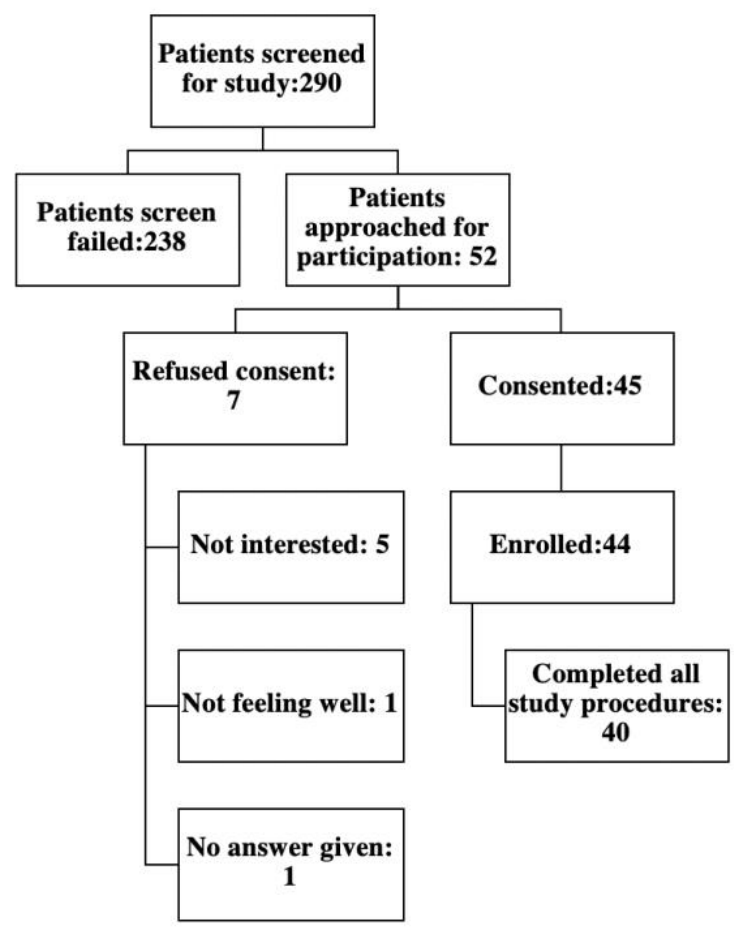

Figure 3: Enrollment of study participants

The majority of enrolled patients were white females $(56.8 \%)$ (Table 1). Eighty-two percent $(n=36)$ of patients enrolled in the study were discharged to home from the EDOU, and the average length of stay in the EDOU was 23.7 hours.

\begin{tabular}{|l|l|}
\hline Gender & $19(43.2 \%)$ \\
Male, n $(\%)$ & $25(56.8 \%)$ \\
Female & $31(70.5 \%)$ \\
Race & $6(13.6 \%)$ \\
White & $2(4.5 \%)$ \\
Black or African American & $5(11.4 \%)$ \\
Asian & \\
Other & $7(15.9 \%)$ \\
Ethnicity & $37(84.1 \%)$ \\
Hispanic or Latino & \\
Non-Hispanic or Latino & $54.0(20.1)$ \\
Average Age (Years) & 53.5 \\
Mean $( \pm$ SD) & $(21,84)$ \\
Median & \\
Min, Max & $30.3(8.7)$ \\
BMI (kg/m $\left.{ }^{2}\right)$ & 27.4 \\
Mean $( \pm$ SD) & \\
Median &
\end{tabular}


\begin{tabular}{l|l|}
\hline Min, Max & $(20.2,61.6)$ \\
\hline \\
Table 1. Participant characteristics
\end{tabular}

We were able to successfully place the wireless wearable biosensors on study participants, connect the sensor to the network, and collect 12 hours of biosensor data $93.2 \%(n=41)$ times. In $5.9 \%(n=3)$ instances, we were not able to successfully connect the sensor to the installed network and the initial biosensor was replaced with a new device. For seven of our enrollments, we also noted that we were unable to connect the tablet to the installed network. We noted that the data server placed under a work desk had been unplugged. We power reset and rebooted the system, which then acquired a signal from established biosensors. When the network was inactive, the established biosensors stored data locally to upload to the server when connectivity was restored. Across all biosensors used in the study, $0.1 \% \quad(\mathrm{SD}=0.2)$ of calculated observations were not uploaded to the server due to network latency.

\subsection{Participant Experience Questionnaire}

After biosensor removal and skin assessment, participants completed the Participant Experience Questionnaire. This questionnaire is comprised of six questions that asked participants to rate their overall experience and comfort level wearing the biosensor during their stay in the EDOU. Overall, 93.2\% of patients reported that they experienced no discomfort during the time that they were wearing the device; $90.9 \%$ of patients said they would wear the device again. Results of the Participant Experience Questionnaire are shown in Table 2.

\begin{tabular}{|l|l|}
\hline $\begin{array}{l}\text { Experienced discomfort } \\
\text { during placement of device }\end{array}$ & None: 100\% $(\mathrm{n}=44)$ \\
\hline $\begin{array}{l}\text { Experienced discomfort } \\
\text { while wearing device }\end{array}$ & $\begin{array}{l}\text { None: } 93.2 \%(\mathrm{n}=41) \\
\text { Minimal: } 2.3 \%(\mathrm{n}=1) \\
\text { Missing data: } 4.5 \% \\
(\mathrm{n}=2)\end{array}$ \\
\hline $\begin{array}{l}\text { Experienced discomfort } \\
\text { during device removal }\end{array}$ & $\begin{array}{l}\text { None: } 59.1 \%(\mathrm{n}=26) \\
\text { Minimal: } 27.3 \% \\
(\mathrm{n}=12)\end{array}$ \\
& $\begin{array}{l}\text { Mild: } 2.3 \%(\mathrm{n}=1) \\
\text { Moderate: } 4.5 \%(\mathrm{n}=2)\end{array}$ \\
& Severe: $2.3 \%(\mathrm{n}=1)$ \\
& $\begin{array}{l}\text { Missing data: } 4.5 \% \\
(\mathrm{n}=2)\end{array}$ \\
\hline Inconvenienced while & None: $93.2 \%(\mathrm{n}=41)$ \\
wearing device & Mild: $2.3 \%(\mathrm{n}=1)$ \\
& $\begin{array}{l}\text { Missing data: } 4.5 \% \\
(\mathrm{n}=2)\end{array}$ \\
\hline
\end{tabular}

\begin{tabular}{|l|l|}
\hline $\begin{array}{l}\text { Power button clicking was } \\
\text { noticeable }\end{array}$ & $\begin{array}{l}\text { No: 95.5\% }(\mathrm{n}=42) \\
\text { Missing data: 4.5\% } \\
(\mathrm{n}=2)\end{array}$ \\
\hline $\begin{array}{l}\text { Would wear the device } \\
\text { again }\end{array}$ & Yes: $90.9 \%(\mathrm{n}=40)$ \\
& No: $4.5 \%(\mathrm{n}=2)$ \\
& Missing data: $4.5 \%$ \\
& $(\mathrm{n}=2)$ \\
\hline
\end{tabular}

Table 2: Patient Experience Questionnaire

\section{Discussion}

This investigation demonstrates that a wireless wearable biosensor that collects physiological data and contextual parameters is feasible to deploy in an EDOU. We additionally demonstrated that patients are accepting of biosensors and are willing to wear them in this setting. One of our most important findings was the high degree of multidisciplinary collaboration needed to deploy a wireless biosensor system in the emergency department. These results suggest that the deployment of a wireless wearable biosensor system in this setting is feasible. Our deployment roadmap may be used by others seeking to use similar technology in hospital settings.

Participants in the study were accepting of the use of cutaneous wireless wearable biosensors during their emergency department stay. They considered the device unobtrusive and comfortable. Participants did not feel inconvenienced by wearing the device, and most would be willing to wear a biosensor during their emergency department stay. Additionally, we were able to train research assistants to apply and connect the biosensor to the wireless network. In the future, patients who enter the emergency department may be able to have the biosensor placed by nursing staff or a medical assistant during their initial triage, either in the waiting room or inside the emergency department. In aggregate, this data suggests that the sensor is easy to operate, and patients who may need supplementary monitoring in the emergency department will wear these devices or similar devices.

The success of this investigation relied on close collaboration with staff from information security, hospital engineering, emergency department operations as well as research staff. We recommend early engagement with hospital information security personnel. Initial deployment considerations such as where to place devices on a hospital network can take time to elucidate. There are advantages in integrating biosensors into existing networks-they allow for easy integration into hospital workflows and allow nascent investigations to understand potential deficiencies in signal transmission. At the same time, integration into a secure hospital network may be 
time-consuming and beyond the scope of an initial feasibility demonstration project. In response, we recommend consideration of alternative data collection and integration measures. These may include installing a separate, independent network in a small portion of the study site or enabling passive data collection from biosensors using low energy Bluetooth or active radiofrequency identification systems. These systems may represent a novel workflow where the isolation of biosensor data allows for a wireless biosensor system to continue to function in the event of network downtime.

In this investigation, we decided, in collaboration with our hospital information security specialists, to install an independent wireless network to accommodate the biosensor. This approach required us to interface with our hospital's engineering group in order to install a simple network consisting of two routers and a series of data servers. Like most hospital emergency departments, we are constrained by space, and the integration of these devices among the ebb and flow of patient volume, nursing and provider workflows was critical. We ultimately feel our solution was advantageous since it allowed us to isolate biosensor data from other clinical data in the emergency department and enabled us to assess for potential disruptions in data transmission from biosensor to network.

\subsection{Implications of this work}

There are several important implications to this work. First, the deployment of wearable biosensors may alter the method in which emergency department's triage and deliver care to patients that traditionally needed a wired monitor. For example, patients with high-risk chief complaints like chest pain, palpitations, or shortness of breath typically require a room in the emergency department or hallway bed with a wired monitor to measure dynamic changes in heart rate, oxygen saturation, or respiratory rate. The potential to expand the footprint within the emergency department in which these patients can be monitored and cared for may allow emergency departments to think about where these patients are roomed (e.g., they could potentially be cared for in spaces that traditionally did not have wired monitors).

Second, independent wireless networks can be installed to cover the emergency department and waiting room. This setup permits wireless, unobtrusive physiological data transmission to be initiated at initial triage of emergency department patients. Current emergency medicine triage practices rely on emergency department staff obtaining vital signs as patients register in the waiting room.
Depending on the emergency severity index (ESI) assigned to the patient, the presence of beds in the emergency department and availability of providers, patients may wait for extended periods in the waiting room. The wireless wearable biosensors could be deployed among waiting room patients to detect potential changes in physiology that may necessitate an "up triage" or potential change in ESI and subsequent rapid entry into the emergency department.

Finally, now that we have established the feasibility of deploying wireless biosensors in the emergency department, our next steps are to understand the impact on clinical operations and care that a wireless biosensor may have in emergency medicine. Although data can be collected on patients in the emergency department, an infrastructure to respond and manage the additional numbers of potential care areas that are enabled through a wireless and unobtrusive biosensor needs to be developed and studied.

\subsection{Limitations and future work.}

This investigation had several limitations. First, we deployed the wearable biosensor in a single, urban, academic emergency department. Our experience with designing and implementing this study may vary in community emergency departments or other hospital locations. Because we wanted to demonstrate that the use of the biosensor system was feasible, we did not study methods in which biosensor data should be integrated into clinical workflow. We also did not test the accuracy of data collected from the biosensor. Finally, we did not consider the cost of installing the infrastructure necessary to utilize the sensor. We installed two routers and a small, off-the-shelf data server to support the flow of biosensor data in the study. Future investigations should include an economic analysis on the financial feasibility of such a system.

In the future, we anticipate investigating operational factors that allow for the integration of wearable biosensor data into emergency department workflows. This is of particular interest to us as emergency department patients may move through various departments in order to obtain imaging studies and other procedures during their emergency department stay. It will be important to understand how biosensors are applied and how consultants are notified of the presence of the sensor. We also plan to understand how the biosensor can be used during the transition from emergency department to inpatient, observation, or outpatient care.

\section{Conclusion}


Overall, this study demonstrated that a wireless wearable biosensor system is a feasible technology to deploy in the emergency department. Participants who wore the biosensor were accepting of the biosensors and reported that it was comfortable; we experienced no serious adverse reactions to the biosensor. We were able to successfully place biosensors and acquire signal $93.2 \%$ of the time. This investigation lays the foundation for future studies that will understand how the biosensor integrates into clinical workflows.

\section{Acknowledgements}

This investigation was funded by Philips Connected Sensing (PI: PRC). PRC is funded by NIH K23DA044874, R44DA051106, The Hans and Mavis Lopater Psychosocial Foundation, and investigatorinitiated grants from e-ink corporation. EWB is funded by NIH R01DA047236. SD is an employee of Philips Connected Sensing.

\section{References}

1. Carreiro S, Chai PR, Carey J, Chapman B, Boyer EW. Integrating Personalized Technology in Toxicology: Sensors, Smart Glass, and Social Media Applications in Toxicology Research. Journal of medical toxicology : official journal of the American College of Medical Toxicology. 2017;13(2):166-172.

2. Peng RC, Zhou XL, Lin WH, Zhang YT. Extraction of heart rate variability from smartphone photoplethysmograms. Comput Math Methods Med. 2015;2015:516826.

3. Chai PR, Carreiro S, Innes BJ, et al. Oxycodone Ingestion Patterns in Acute Fracture Pain With Digital Pills. Anesthesia and analgesia. 2017;125(6):2105-2112.

4. Melvin E, Cushing A, Tam A, Kitada R, Manice M. Assessing the use of BreatheSmart ${ }^{\circledR}$ mobile technology in adult patients with asthma: a remote observational study. BMJ Open Respir Res. 2017;4(1):e000204.

5. Sharma A, Harrington RA, McClellan MB, et al. Using Digital Health Technology to Better Generate Evidence and Deliver Evidence-Based Care. J Am Coll Cardiol. 2018;71(23):2680-2690.

6. Claudio D, Velázquez MA, Bravo-Llerena W, Okudan GE, Freivalds A. Perceived Usefulness and Ease of Use of Wearable Sensor-Based Systems in Emergency
Departments. IIE Transactions on Occupational Ergonomics and Human Factors. 2015;3(3-4):177-187.

7. Adapa A, Nah FF-H, Hall RH, Siau K, Smith SN. Factors Influencing the Adoption of Smart Wearable Devices. International Journal of Human-Computer Interaction. 2018;34(5):399-409.

8. Li T, Divatia S, McKittrick J, Moss J, Hijnen NM, Becker LB. A pilot study of respiratory rate derived from a wearable biosensor compared with capnography in emergency department patients. Open Access Emerg Med. 2019;11:103-108.

9. Chai PR. Wearable Devices and Biosensing: Future Frontiers. Journal of medical toxicology : official journal of the American College of Medical Toxicology. 2016;12(4):332-334.

10. Dunn J, Runge R, Snyder M. Wearables and the medical revolution. Personalized Medicine. 2018;15(5):429-448.

11. Datatrack. https://datatrack.com. Accessed June, 2020. 